\title{
The Effect of Exercise on Pulpal and Gingival Blood Flow in Physically Active and Inactive Subjects as Assessed by Laser Doppler
}

\author{
E.C. Lobo ${ }^{1}$, S.M.T. Nguyen ${ }^{1}$ and M.A. Pogrel ${ }^{2}$ * \\ ${ }^{I}$ Department of Oral and Maxillofacial Surgery University of California San Francisco \\ ${ }^{2}$ Department of Oral and Maxillofacial Surgery
}

\begin{abstract}
The effects of exercise on pulpal and gingival blood flow are undefined. The autonomic nervous system response suggests that they could increase or decrease with exercise, and they may be independent of each other. This study attempts to answer these questions.

Materials and Methods: 15 "physically active" subjects and 15 "physically inactive" subjects exercised on a treadmill. Laser Doppler recordings of pulpal and gingival blood flow were taken before and after exercise.

Results: There was a mean increase of approximately $50 \%$ in both pulpal (range $-66 \%-+509 \%)$ and gingival (-72\%$+1022 \%$ ) blood flow after exercise, with wide variations, with no significant differences between the two groups of subjects.

Conclusion: Exercise could increase pulpal and/or gingival blood flow as part of the overall increase in cardiac output with exercise, or could decrease pulpal and/or gingival blood flow due to diversion of blood to the somatic muscles during exercise. This study suggests that the former physiological phenomenon usually takes place at both sites, though there were some contradictory results.
\end{abstract}

Keywords: Pulp blood flow, Gingival Blood Flow, Laser Doppler.

\section{INTRODUCTION}

The blood supply to the teeth and adjacent gingiva is known to be under autonomic control, as in other parts of the body. However, different control mechanisms appear to apply in these two adjacent areas. The blood supply to the pulp itself appears to be under cholinergic control [1], whereas the blood supply to the gingiva appears to be under non adrenergic, non cholinergic, control [2]. Therefore different autonomic stimuli may induce different responses in the vascularity of the pulp and adjacent gingiva [3-5]. At the present time, there is minimal knowledge of the effects of exercise on pulpal and gingival blood flow. This is because not only are the pulpal and gingival vascularity under different autonomic control, but the different ectodermal and mesenchymal origins of these tissues could affect autonomic responses. For example pulpal and gingival blood flow could increase with exercise in the same way that the blood supply to the somatic musculature increases with exercise, or the blood supply could decrease in the same way as the blood supply to the gastrointestinal tract decreases with exercise, as blood is channeled to the somatic musculature [6,7]. This could be of relevance in subjects taking prolonged exercise. Although there appear to be no specific reports in the literature of dental ischemia or hyperemia in athletes, there are

*Address correspondence to this author at the Department of Oral and Maxillofacial Surgery University of California San Francisco Box 0440, 521 Parnassus Ave., Room C522 San Francisco, CA 94143-0440;

Tel: (415) 476-8226; Fax: (415) 476-6305; E-mail: tony.pogrel@ucsf.edu many anecdotal reports of dental throbbing and pain with exercise [8], and also reports of oral pain with marathon running [9]. These could be due to changes in vascularity.

It is not known whether there is any difference in the effect on pulpal and gingival blood flow with exercise, between those who exercise on a regular basis and those who do not. The purpose of this study was to determine the effect of exercise on pulpal and gingival blood flow in subjects who exercised regularly and those who did not, utilizing a laser Doppler monitor with a $1 \mathrm{~mm}$ needle point laser probe.

\section{MATERIALS AND METHODS}

This study utilized adult student volunteers for whom institutional human research approval had been obtained. The study involved 15 subjects who exercised regularly for 30 minutes or more at least 3 times a week during the past six months (the physically active group) and 15 subjects who did not partake in regular exercise (the physically inactive group). All subjects were in good general health and between the ages of 18 and 28. Exclusion criteria included any history of smoking, diabetes or cardiovascular disease, and because the upper right central incisor was used for this study, an additional exclusion criteria was a restored or nonvital upper right central incisor. Subjects with periodontal disease or undergoing orthodontic treatment were also excluded from the study. The Body Mass Index (BMI) was calculated for each subject to evaluate whether it had any relationship to pulpal or gingival blood flow. The heart rate and blood pressure were recorded before and after exercise to assess the 


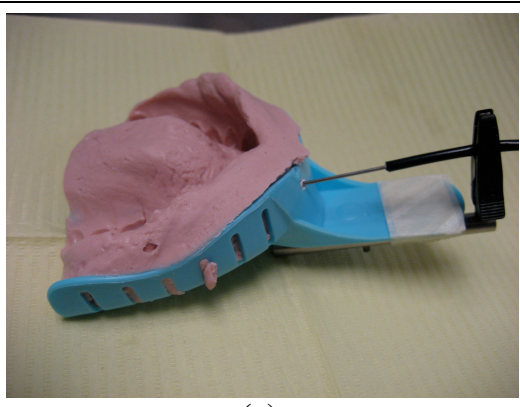

(a)

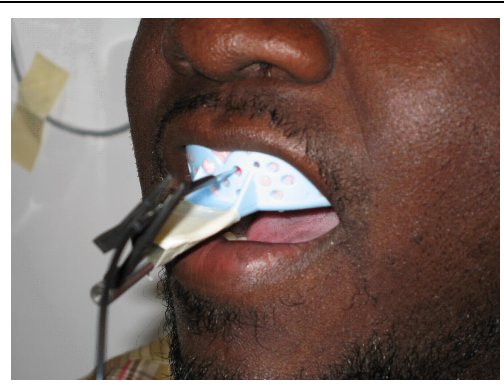

(b)

Fig. (1). Upper alginate impression with needle probe pressed through a hole (a) to touch the crown followed by the adjacent gingival (b).

fitness to exercise, to ensure that subjects reached $80 \%$ of their maximum heart rate, and to monitor the blood pressure increase with exercise. The vitality of the upper right central incisor was confirmed by means of an electric pulp tester.

Subjects first had an alginate dental impression taken of the maxilla, utilizing a perforated tray to include the labial surfaces of both upper central incisors and associated gingiva. The alginate was mixed thinly so that there was no pressure, or blanching, of the gingiva. Following removal of the tray from the mouth, the alginate impression was kept moist, to avoid shrinkage and distortion, and two holes were placed for a $1 \mathrm{~mm}$ diameter needlepoint laser Doppler probe to be inserted, to touch sequentially the center of the visible crown of the upper right central incisor and the adjacent gingiva, $6 \mathrm{~mm}$ superior to the center of the gingival margin of the upper right central incisor (Fig. 1). Care was taken to ensure that alginate did not obstruct the probe tip. Utilizing the Periflux PF3 Laser Doppler Perfusion Monitor (PeriMed, Stockholm, Sweden), and a $1 \mathrm{~mm}$ needlepoint probe, recordings were made of the pulpal blood flow in the upper right central incisor and the adjacent gingiva, $6 \mathrm{~mm}$ from the gingival margin. Results were recorded in the arbitrary perfusion units of the laser Doppler perfusion monitor (Fig. 2). Subjects then underwent exercise on a treadmill until they reached $80 \%$ of their maximum heart rate (maximum heart rate $=208$ minus (0.7 times age in years $)$ ) [10]. Once the subject reached $80 \%$ of maximum heart rate, they continued on the treadmill fora total of 5 minutes. Five minutes was chosen as the exercise time, after discussion with a university fitness instructor, in order to elevate the heart rate to a steady state and maintain it there for a moderate length of time but not to continue the exercise for so long that it could be exhausting, or even potentially dangerous.

At the end of this period, the pulpal blood flow from the upper right central incisor and adjacent gingival blood flow were recorded again, in the arbitrary perfusion units of the laser Doppler. In order to accomplish this, the alginate impression was re-placed in the mouth and the laser Doppler probe reinserted through the same holes as pre-exercise to touch sequentially the crown of the tooth and the gingiva respectively. Since the probe tip was firmly held in the alginate impression, which was firmly fixed to the subject's teeth, motion artifact should be eliminated. The pulpal blood flow was recorded first (within 20 seconds of termination of exercise) followed by the gingival blood flow as soon as the pulpal blood flow had stabilized. When not in use, the alginate impression was stored in a sealable plastic bag with

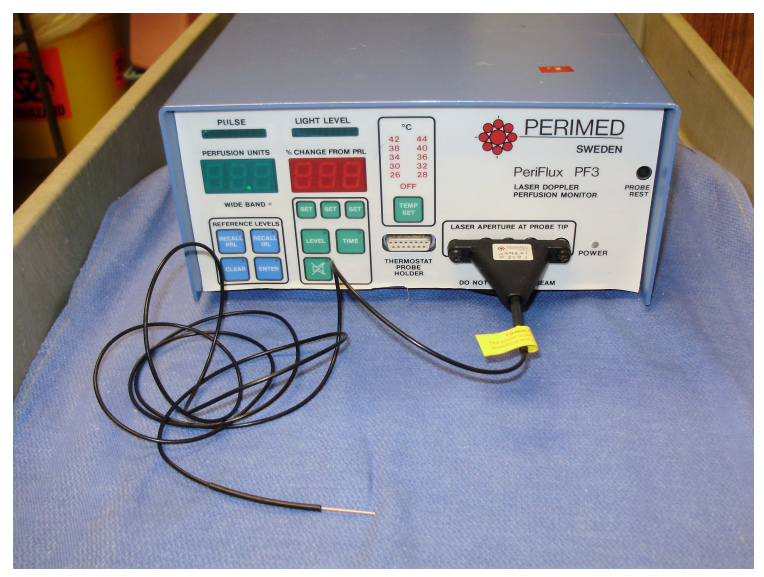

Fig. (2). The Periflux PF3 laser Doppler with $1 \mathrm{~mm}$ needlepoint probe.

moist gauze to minimize any distortion. Prior to the laser Doppler being used on each occasion, it was zeroed utilizing a stationary object, and calibrated utilizing a proprietary motility standard.

Statistical analysis was performed utilizing a paired t-test, looking at pre-exercise Pulpal Blood Flow vs postexercise Pulpal Blood Flow and pre-exercise Gingival Blood Flow vs. post-exercise Gingival Blood Flow on both groups of subjects (those who took regular exercise and those that did not). A p value of 0.05 or less was considered significant.

\section{RESULTS}

The study group consisted of 30 healthy subjects aged between 18 and 28 of whom half took regular exercise consisting of at least 30 minutes 3 times a week (the physically active group), and the other half did not (the physically inactive group). There were an equal number of males and females. In all cases post exercise Laser Doppler readings on the pulp were taken within 20 seconds of terminating the exercise and the gingival recordings were taken within 50 seconds of terminating the exercise. It took about 10 seconds for the Laser Doppler readings to stabilize and then the probe tip was left in place for a further 20 seconds and an average reading was recorded. A total recording time of 1 minute post exercise was selected since one needed to cease the exercise, seat the patient in a chair, and accurately seat the dental impression. However, one did not want to wait long enough that the autonomic changes had time to reverse themselves. One minute was the shortest standardized time in which this could be achieved. 
Table 1. Laser Perfusion Units Before and after Exercise for each or the 30 Subjects

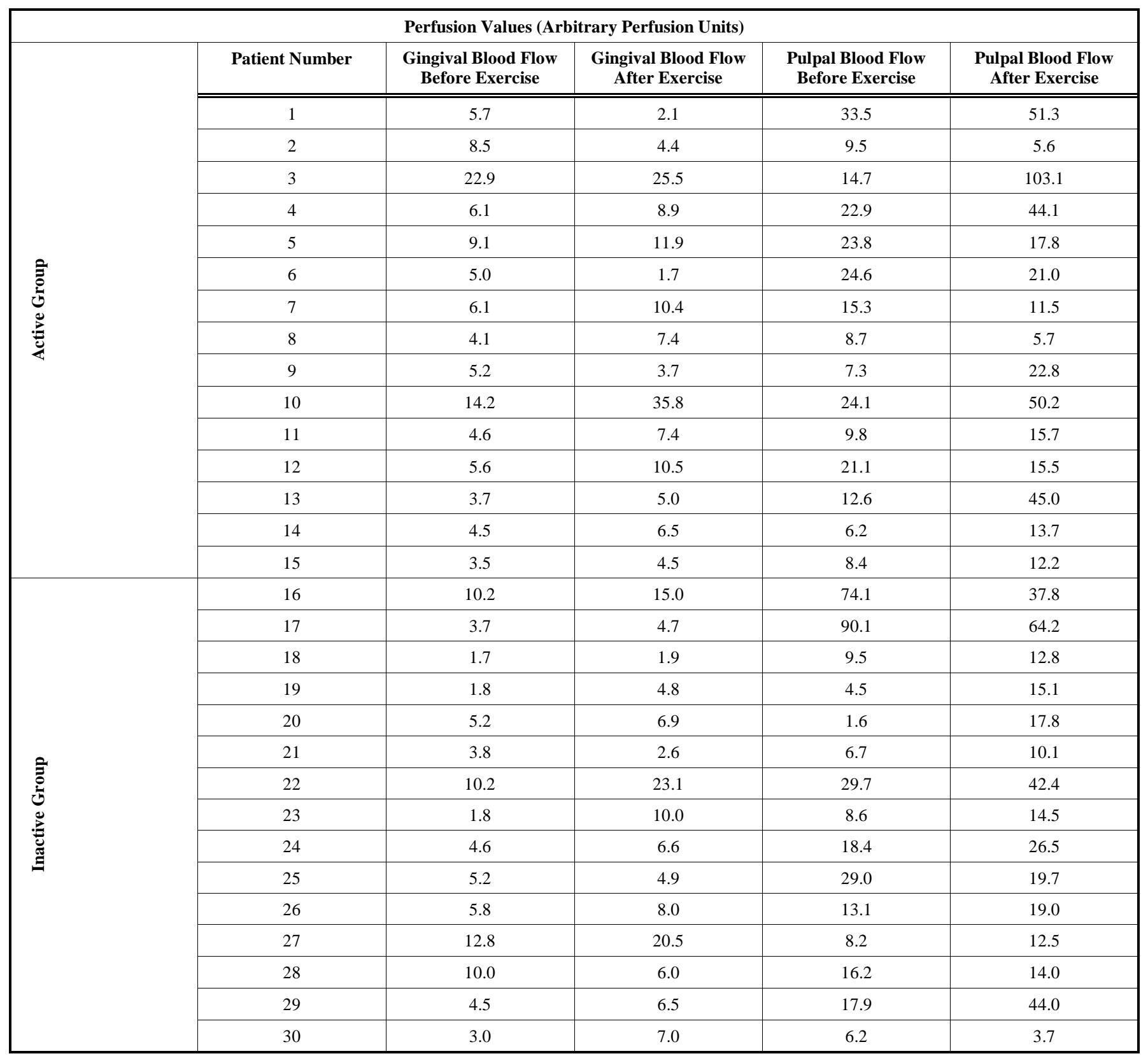

For the "physically active" group (15 subjects):

- Eight showed an increase in pulpal blood flow and gingival blood flow after five minutes exercise.

- Two showed a decrease in pulpal blood flow and gingival blood flow.

- Three had an increase in pulpal blood flow but a decrease in gingival blood flow

- Two had a decrease in pulpal blood flow but an increase in gingival blood flow.

For the "physically inactive" group (15 subjects):

- Nine showed an increase in pulpal blood flow and gingival blood flow with acute exercise.
- Two had a decrease in pulpal blood flow and gingival blood flow.

- Three had an increase in pulpal blood flow but a decrease in gingival flow.

- One subject had a decrease in pulpal blood flow and an increase in gingival blood flow.

If these two groups are combined, the overall changes were:

- Seventeen (57\%) had an increase in pulpal blood flow and gingival blood flow after exercise.

- Four (13\%) had a decrease in pulpal blood flow and gingival blood flow. 


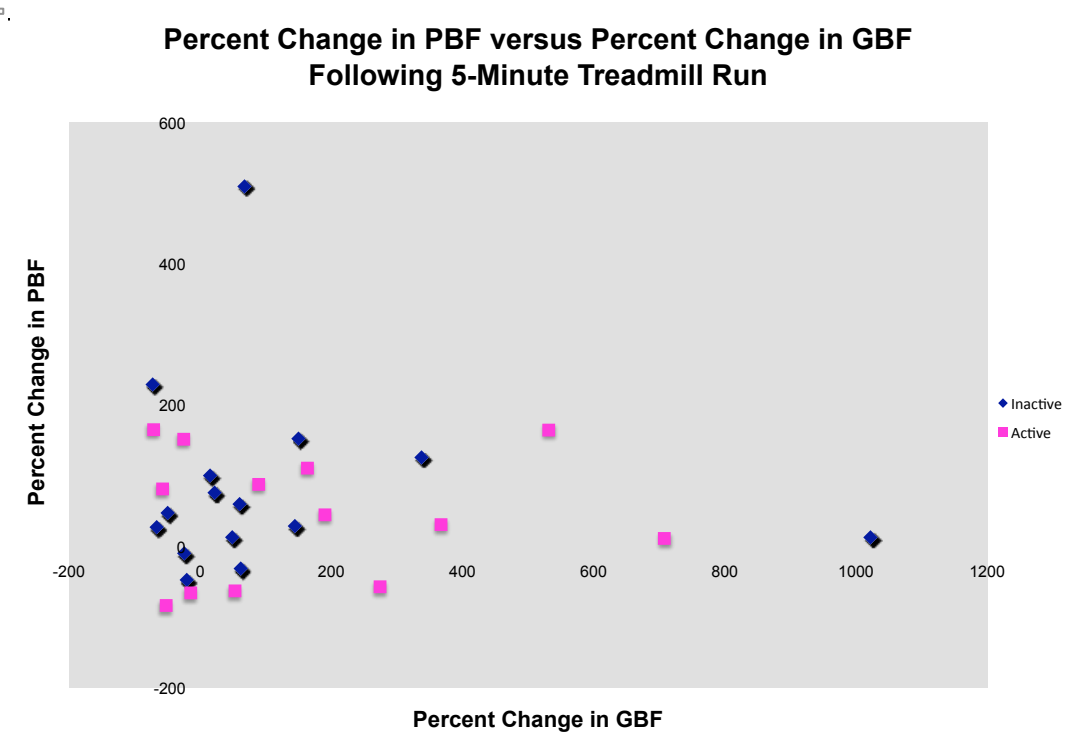

Fig. (3). Scattergram showing the effect of exercise on pulpal and gingival blood flow in physically active and non active subjects.

- $\quad$ Six $(20 \%)$ had an increase in pulpal blood flow but a decrease in gingival blood flow.

- $\quad$ Three $(10 \%)$ had a decrease in pulpal blood flow but an increase in gingival blood flow.

The actual Perfusion Units are shown in Table 1. The results are summarized as percentage change in the scattergram in Fig. (3). Although the units on the laser Doppler are arbitrary perfusion units, the mean increase in pulpal and gingival blood flow with exercise (in those who exercised regularly and those who didn't) was $50 \%$, though there were wide variations (range for pulpal blood flow $-56 \%-+509 \%$ and for gingival blood flow $-72 \%-+1022 \%$ ).

Changes in pulpal blood flow occurred independently of changes in gingival blood flow. Exercise appears to increase pulpal blood flow in most volunteers, but its effect on gingival blood flow is less consistent. Subjects were more likely to have an increase in pulpal blood flow than gingival blood flow. Pulpal blood flow increased with exercise in $77 \%$ of the total subjects, in $73 \%$ of the physically active subjects, and in $80 \%$ of the physically inactive subjects. This reached statistical significance $(\mathrm{p}=0.02)$ for the combined groups (those who took exercise and those that did not) but it was the only one of the paired t tests which did achieve statistical significance.

The effect of exercise on gingival blood flow, was not as consistent. $67 \%$ of the total (67\% of the physically active group, and $67 \%$ of the physically inactive group) displayed an increase in gingival blood flow with exercise. No statistically significant alterations for gingival blood flow were found before and after exercise for either group of subjects, or for the subjects combined.

There were no significant associations between pulpal or gingival blood flow and gender or Body Mass Index (BMI) at baseline or after exercise for either group of subjects.

Any recorded decreases in pulpal or gingival blood flow were by a mean of $30 \%$, though again there were variations (range $-6 \%--84 \%$ for pulpal blood flow and $-14 \%--70 \%$ for gingival blood flow) with no statistically significant differences.

In all subjects, heart rate increased during this study, and in fact the recordings did not commence until subjects had reached a calculated $80 \%$ of maximum heart rate. After this, however, heart rate was not monitored again until the end of the study. Similarly, blood pressure was recorded at the beginning and end of the exercise primarily for subject safety. Direct correlations were not sought, but it is true that in all cases heart rate did increase during the exercise, and in most (but not all) cases blood pressure also increased between the start and end of the exercise.

\section{DISCUSSION}

From this study, exercise, known to increase heart rate and cardiac output, usually increased the blood supply to the upper central incisor tooth and associated gingiva. This occurred in both those who regularly took exercise and those that did not, though the pulpal vascularity tended to increase more in those who did not take regular exercise. These results were not statistically significant however. Blood flow responses were inconsistent, however, and in a number of subjects there were decreases in either pulpal and/or gingival blood flow.

With no evident physical abnormalities in either the tooth or gingiva, the varied outcomes are presumably a physiological response that may lie in the different embryonic origin of the two tissues. The pulp is mesenchymal in origin and therefore blood flow might be expected to increase with exercise. The gingiva, on the other hand, is ectodermal, so blood flow may depend on the individual's response to exercise. Although there is an increase in cardiac output, blood flow may be diverted to other more essential organs.

The lack of significant correlations may also indicate the presence of other parameters affecting the pulpal and gingival blood flow, independent of the autonomic nervous system. 
It is known that Laser Doppler recordings can be subject to interference and artifact. Studies have shown that adjacent structures can interfere with Laser Doppler recordings [11, 12], but our use of an alginate filled impression tray should help to eliminate this artifact, together with probe positioning at least $6 \mathrm{~mm}$ from the gingival margin. Although our results show wide variations, the readings were stable over the 20 second recording period, and are consistent with other studies. It is felt that the possibility of artifact was minimized, and that the results, with the wide variations, are accurate, and represent the relative blood flow. The fact that gingival recordings were taken about 50 seconds after the pulpal recordings is not felt to represent a significant factor in the results.

Published literature on this subject is limited, but laser Doppler flowmetry has proved to be an acceptable method of assessing pulpal and gingival blood flow, as well as pulpal vitality [5, 13-16]. The specific technique of using an alginate impression to stabilize and standardize the laser Doppler and reduce artifact when recording pulpal and gingival blood flow has been previously evaluated by one of the present authors [17]. Literature also exists on the nervous factors regulating pulpal and gingival blood flow [2, 3]. A study on exercise and pulpal blood flow in diabetic rats showed that pulpal blood flow decreased with exercise, but that exercise training can prevent the reduction [18].

Only one study appears to have examined pulpal and gingival blood flow with exercise in humans, utilizing a laser Doppler [19]. This study measured blood flow to 17 upper incisors and associated attached gingiva in 10 male subjects, as compared to 30 subjects in the current study. Each subject pedaled on a bicycle until the heart rate rose at least $50 \%$, after which the exercise was continued for a further $1 \frac{1}{2} \mathrm{~min}$ utes. With the pedal cycle exercise the pulpal blood flow increased a mean of $38 \%$ but with a wide range from $-100 \%$ to $+200 \%$. To the gingiva, the blood flow increased a mean of $65 \%$ with a range from $-6 \%$ to $+300 \%$. The heart rate increased by a mean of $54 \%$, but with great variation and with no direct correlation between the increased heart rate and change in pulpal or gingival blood flow. The pulpal and gingival blood flow with exercise increased in most subjects, although there was wide variability. In this respect, the figures from this previously published study are very similar to those obtained in the present study.

\section{CONCLUSION}

Although one can never totally rule out artifact with Laser Doppler we feel that these results are accurate, and reflect wide variations in pulpal and gingival blood flow with exercise, in both physically active and inactive subjects. In the majority of cases the pulpal and gingival blood flow in- creased with exercise in both physically active and inactive subjects.

\section{ACKNOWLEDGEMENT}

Internal Departmental Funding Only.

\section{CONFLICTS OF INTEREST}

\author{
None decleard.
}

\section{REFERENCES}

[1] Aars H, Brodin P, Andersen E. A study of cholinergic and betaadrenergic components in the regulation of blood flow in the tooth pulp and gingiva in man. Acta Physiol Scand 1993; 148: 441-7.

[2] Aars H, Gazelius B, Edwall L, Olgart L. Nervous control of blood flow in the human tooth. Acta Physiol Scand 1990; 140: 7A.

[3] Aars H, Gazelius B, Edwall L, Olgart L. Effects of autonomic reflexes on tooth pulp blood flow in man. Acta Physiol Scand 1992; 146: 423-9.

[4] Costill D, Wilmore JH. Physiology of Sport and Exercise. $3^{\text {rd }}$ ed. Champaign Illinois: Human Kinetics Publishers 2004; vol. 50: pp. 566-602.

[5] Ramsay DS, Artun J, Martinen SS. Reliability of pulpal blood-flow measurements utilizing laser Doppler flowmetry. J Dent Res 1991; 70: $1427-30$.

[6] Olgart L. Neural control of pulpal blood flow. Crit Rev Oral Biol Med 1996;7: 159-71

[7] Thomas GD, Segal SS. Neural control of muscle blood flow during exercise. J Appl Physiol 2004; 97: 731-8.

[8] Kemppainen P, Pertovaara A, Huopaniemi T, Johansson G, Karonen SL. Modification of dental pain and cutaneous thermal sensitivity by physical exercise in man. Brain Res 1985; 360: 33 40 .

[9] Steel B. Running pain. Br Dent J 2010; 208 (9): 380.

[10] Arnall D. ACSM's guidelines for exercise testhing and prescription. 2000; available from http:// jan.ucc.nau.edu/daa/ACSMGuidelines/

[11] Soo-ampon S, Vongsavan N, Soo-ampon M, Chuckpaiwong S, Matthews B. The sources of laser Doppler blood-flow signals recorded from human teeth. Arch Oral Biol 2003; 48: 353-60.

[12] Vongsavan N, Matthews B. Experiments in pigs on the sources of laser Doppler blood-flow signals recorded from teeth. Arch Oral Biol 1996; 41: 97-103.

[13] Dodson TB, Bays RA, Neuenschwander MC. Maxillary perfusion during Le Fort I osteotomy after ligation of the descending palatine artery. J Oral Maxillofac Surg 1997; 55: 51-5.

[14] Gazelius B, Olgart L, Edwall B. Restored vitality in luxated teeth assessed by laser Doppler flowmeter. Endod Dent Traumatol 1988; 4: $265-8$.

[15] Gazelius B, Olgart L, Edwall B, Edwall L. Non-invasive recording of blood flow in human dental pulp. Endod Dent Traumatol 1986; 2: 219-21.

[16] Olgart L, Gazelius B, Lindh-Stromberg U. Laser Doppler flowmetry in assessing vitality in luxated permanent teeth. Int Endod J 1988; 2: 300-6.

[17] Ahn J, Pogrel MA. The effects of 2\% lidocaine with 1:100000 epinephrine on pulpal and gingival blood flow. Oral Surg Oral Med Oral Pathol Oral Radiol Endod 1998; 197-202.

[18] Amatyakul S, Chakraphan D, Chotipaibulpan S, Patumraj S. Role of exercise training on pulpal blood flow in diabetic rats. Clin Hemorheol Microcirc 2006; 34: 295-301.

[19] Watson AD, Pitt Ford TR, McDonald F. Blood flow changes in the dental pulp during limited exercise measured by laser Doppler flowmetry. Int Endod J 1992; 25: 82-7. 